Revue d'histoire de l'Amérique française

ARS REVUE D.HISTOIRE DE L'AMÉRIQUE FRANÇAISE

\title{
Monseigneur Antoine Racine et la question universitaire canadienne (1875-1892) (suite)
}

\section{Germain Lavallée}

Volume 12, numéro 2, septembre 1958

URI : https://id.erudit.org/iderudit/301904ar

DOI : https://doi.org/10.7202/301904ar

Aller au sommaire du numéro

Éditeur(s)

Institut d'histoire de l'Amérique française

ISSN

0035-2357 (imprimé)

1492-1383 (numérique)

Découvrir la revue

Citer cet article

Lavallée, G. (1958). Monseigneur Antoine Racine et la question universitaire canadienne (1875-1892) (suite). Revue d'histoire de l'Amérique française, 12(2), 247-261. https://doi.org/10.7202/301904ar d'utilisation que vous pouvez consulter en ligne. 


\title{
MONSEIGNEUR ANTOINE RACINE
}

\author{
ET \\ LA QUESTION UNIVERSITAIRE CANADIENNE \\ $(1875-1892) *$ \\ (suite) \\ Chaprtre VI \\ DECRETS SUR DECRETS ET... \\ LA LUTTE N'EN EST QUE PLUS ENGAGEE
}

Deux décrets de Rome - Arrivée du Délégué Apostolique, Mgr Smeulders - T.-E. Hamel, Supérieur de la Succursale Rome distribue des honneurs ecclésiastiques - Mgr Fabre veut-il gagner Mgr Racine?

Ce que l'Archevêque de Québec attendait de Rome en février 1882, c'était sans doute un nouveau décret, appuyant les décisions précédentes. Le document ne manquera pas de venir. ${ }^{25}$ Comme on pouvait s'y attendre, le nouveau décret, 27 avril 1882, indisposa le clergé de Montréal qui voulait toujours une université indépendante de Laval. ${ }^{26}$ Il indisposa également quelques évêques. $^{27}$ Les amis de Laval n'en étaient pas moins contents: ils songèrent à envoyer au Saint-Père une adresse de remerciements et à la fois une protestation du dévouement de l'Université Laval et de son obéissance au Saint-Siège. ${ }^{28}$ Mgr Racine se montra très enthousiaste et déclara sa grande joie de signer l'adresse de remerciements au $\mathrm{S}$. Père pour les derniers décrets. Il espérait également que les Evêques en feraient autant. ${ }^{29}$ En quoi il se

*Voir RHAF, XII - No 1 - (juin 1958) : 80-107. A-1, F-1.

25 Mgr Taschereau au Cardinal Siméoni, 24 octobre 1882, AAS, XIII,

26 Mémoire envoyé par le Recteur aux Evêques de la Province, p. 9, AAS, XIII, B-1, A-1.

27 Ibid, 10 .

28 L'abbé P. Méthot à Mgr Racine, 3 mars 1882, AAS, XIII, B-1, A-2.

$29 \mathrm{Mgr} A$. Racine à l'abbé P. Méthot, 4 mars 1882 , Un. 136 - No AH. 
trompait. Le même jour, il recevait de Mgr Langevin un télégramme l'avertissant qu'il ne signerait pas l'adresse sans la discuter à l'assemblée des Evêques. Indigné, Mgr A. Racine refuse de donner une réponse à son confrère de Rimouski. ${ }^{30}$

L'ensemble des Evêques de la Province prennent le parti d'envoyer au Cardinal Siméoni, Préfet de la Propagande, une lettre collective où on le supplie de protéger la Succursale de Montréal, contre les attaques auxquelles elle est soumise. ${ }^{31}$ Cette lettre porte les signatures de l'archevêque de Québec ainsi que celles des Evêques de Montréal (Mgr Fabre), de Sherbrooke et de St-Hyacinthe. Sur les entrefaites une nouvelle parvient à Mgr de St-Hyacinthe: les adversaires de la Succursale ont envoyé à Rome une requête pour que défense soit faite au Séminaire de Québec de bâtir à Montréal. Mgr Moreau ajoute: "C'est le comble de la démence » que de demander pareille chose. ${ }^{32}$ Le Cardinal Siméoni rassure de nouveau l'Archevêque et les Evêques signataires de la lettre collective: "Comme le décret de cette S.C. en 1876 et les deux décrets confirmatifs du même, émanés du $S$. Père sont en pleine vigueur, je conviens avec Votre Grandeur que les exceptions de nullité de ce décret ne méritent aucune considération. ${ }^{33}$ Puis, le 27 février 1883, Léon XIII veut bien encore condescendre à envoyer au Canada français un nouveau décret qu'on peut résumer en ces termes: comme l'Université Laval et sa Succursale établie à Montréal par l'autorité apostolique se trouvent depuis longtemps en butte à de grandes difficultés à cause des discussions qu'on a soulevées, le S. Père demande $1^{\circ}$ qu'on s'en tienne au décret de $1876,2^{\circ}$ à la constitution, i.-e. à la charte civile, $3^{\circ}$ le $\mathrm{S}$. Père défend de tramer quoi que ce soit contre l'Université Laval, $4^{\circ}$ de faire tout le possible pour son expansion. ${ }^{34} \mathrm{Mgr}$ Taschereau, on le comprend,

30 Mgr A. Racine à Mgr Taschereau, 4 mars 1882, Un. 136 - No AH. F-1.

31 Lettre collective au Card. Siméoni, 24 octobre 1882, AAS, XIII, A-1, B-1, A-2.

$32 \mathrm{Mgr}$ Taschereau à Mgr A. Racine, 23 novembre 1882, AAS, XIII, B-1, A-2.

33 Le Cardinal Siméoni à Mgr Taschereau, 27 novembre, AAS, XIII, 34 Décret du 27 février 1883, AAS, XIII, B-1, A-2. 
réagit on ne peut mieux devant le nouveau document: «Voilà un décret fort sérieux. ${ }^{35}$ Il ne reste plus qu'à combiner un petit jeu d'adresse pour gagner l'Evêque de Montréal à sévir contre l'Ecole de Médecine. L'Archevêque écrit à Mgr de Sherbrooke:

Il faut tâcher maintenant de prévaloir sur $\mathrm{Mgr}$ Fabre pour l'engager à agir vigoureusement envers l'Ecole de Médecine. Que dirait V.G. si par un heureux hasard bien convenu d'avance entre V.G. et Mgr Moreau, je rencontrais mes deux collègues pour en conférer ensemble? Je prépare un amendement où j'explique chacune des parties du décret et les devoirs qui en résultent pour tous, Evêques, prêtres, religieux, écrivains, journalistes, etc.

Je veux mettre les points sur les $i^{36}$

Malgré toutes ces démarches de l'Archevêque, Mgr A. Racine s'impatiente; les choses languissent, à ce qu'il lui semble; elles ne prennent pas le chemin des réalités; il s'en explique à T.-E. Hamel:

Je n'ai jamais admiré et je n'admire pas un Général en chef qui donne à son adversaire le temps et l'avantage de se fortifier pendant deux mois.

Puisque le Pape décidait lui-même de la question de l'opportunité, il était évident que son désir était de nous faire agir promptement. Mais que voulez-vous que j'y fasse ? A l'impossible personne n'est tenu. .......

Est-ce qu'il (Mgr Taschereau) ne peut pas comprendre que le délai est une victoire pour les adversaires ?... A quoi cela aboutira-t-il pratiquement si l'Ecole de Médecine reste affiliée (à l'Université Victoria) ? Si l'Hôtel-Dieu de Montréal ouvre ses portes aux professeurs de l'Ecole et les ferme aux professeurs de Laval ? Un décret, c'est très bien, mais il faut le faire exécuter; sinon, vous détruisez l'autorité au lieu de l'affermir. Tout ceci prouve que je suis de mauvaise humeur, comme à mon départ de Québec en janvier dernier. ${ }^{37}$

35 $\mathrm{Mgr}$ Taschereau à Mgr Racine, 20 mars 1883, AAS, XIII, B-1, A-3. 36 Ibid.

37 Mgr A. Racine à T.-E. Hamel, 7 avril 1883, Un. 138 - No H. 
Quelques semaines plus tard, Mgr Racine revient sur le sujet; il continue de se plaindre de la lenteur de l'Archevêque: "Il vaut mieux ne pas recevoir un décret, quelque serré qu'il soit, si ce décret n'est pas exécuté dans toute sa teneur $\gg^{38}$ Mais l'Archevêque va bientôt agir; il rédige un « rapport de nos conférences et correspondances avec les membres de l'Ecole de Médecine de Montréal . $^{39}$ Dans une lettre au Dr d'Orsonnens, Mgr Taschereau précise:

L'Ecole étant ainsi jugée et déclarée rebelle à l'autorité religieuse, il s'en suit comme conséquences: a) que la communauté de l'Hôtel-Dieu de Montréal est libre de toute obligation envers la dite Ecole; b) qu'aucun catholique ne peut plus en conséquence faire partie de la dite Ecole ou en fréquenter les cours, et que les professeurs et les élèves ne peuvent être admis aux sacrements de l'Eglise; c) que l'ordonnance de Mgr de Montréal, contre laquelle l'Ecole en a appelé aux Evêques de la Province, est maintenue. ${ }^{40}$

C'était l'excommunication pour l'Ecole et ses membres, (professeurs et élèves) sans autre forme de procès.

Cette fois Rome va protester contre cet emploi des peines ecclésiastiques. C'est aller trop loin. "Suspende omnia », prononce un télégramme adressé par le Cardinal Siméoni à l'Archevêque de Québec. Mgr $\mathrm{A}$. Racine fait observer que c'est «à cause de la question de justice soulevée par l'Ecole ». A Québec on s'énerve, on veut fermer les portes de Laval en septembre. Mgr de Sherbrooke les invite à ne pas perdre la tête:

Quant à fermer à l'automne les cours à Québec et à Montréal, je suis loin d'être de cet avis. Le St-Siège n'aura pas cette mesure extrême pour agréable. D'ailleurs il sera toujours temps de faire cela, lorsque le Commissaire (délégué apostolique) sera sur les lieux, et qu'il aura été prévenu de votre détermination. ${ }^{11}$

38 Id. à id., 30 avril 1883, Un. 138 - No AD.

39 On peut lire ce rapport, dans l'appendice IV de notre présent travail; AAS, XIII, A-1, F-1.

40 Copie d'une lettre de Mgr Taschereau au Dr d'Orsonnens, 25 juin 1883, AAS, XIII, A-1, F-1. Cette copie est certifiée conforme à l'original par l'abbé C.-A. Marois, secrétaire.

41 Mgr Racine à T.-E. Hamel, 28 août 1883, Un. 139 - No AP. 
Voilà qui avait de quoi relever le courage des Montréalais. Le «Suspende omnia », l'envoi par Rome d'un Commissaire apostolique pour étudier le problème, tout cela permettait un peu, beaucoup d'espoir. "Il paraît que les avocats veulent aussi avoir une faculté de droit indépendante. La bataille va être terrible », d'écrire Mgr Taschereau à l'Evêque de Sherbrooke.43 Le calme Mgr Fabre, dans ses souhaits à Mgr Racine pour l'année 1884 qui va commencer, exprime le désir que la paix descende sur ce triste problème. Il annonce en même temps que le Commissaire envoyé par Rome sera Mgr Smeulders. ${ }^{44}$

Le Commissaire consulte secrètement Mgr Racine, sur les besoins financiers de l'Université qui cherche du secours:

Je me fais un devoir de soumettre en confidence secrète et réservée à l'examen de Votre Grandeur un projet de l'Université Laval qui m'a été présenté par Mgr Benjamin Pâquet. Je vous prie, Monseigneur, de me le renvoyer au plus tôt possible, accompagné des avis et observations que vous jugerez à propos de faire afin que je puisse en juger avec pleine connaissance de cause. Vous direz librement et franchement ce que vous en pensez, Monseigneur. Seulement je vous engage à garder l'affaire en secret. $^{45}$

Pour Mgr A. Racine le délégué de Rome n'agit pas très sérieusement; en réalité, serait-il déterminé à ne rien régler ? Egalement «en confidence secrète et réservée », l'Evêque de Sherbrooke adresse à Mgr Taschereau une copie de la lettre du délégué, puis ajoute:

$\mathrm{Au}$ lieu de proposer à l'attention des Evêques deux ou trois projets, et surtout de nous dire s'il est convaincu lui-même, que l'Université a besoin d'une aide efficace, Son Excellence se contente de nous adresser une copie de la lettre de Mgr Pâquet.

$43 \mathrm{Mgr}$ Taschereau à Mgr A. Racine, 10 novembre 1883, AAS, XIII, A-1, F-1.

$44 \mathrm{Mgr}$ Fabre à Mgr A. Racine, 26 décembre 1883, AAS, VII, A-1, G-10.

$45 \mathrm{Mgr}$ H. Smeulders à Mgr A. Racine, 20 mars 1884, AAS, XIII, B-1, A-3; Un. 141 - No D. Le prélat envoyé par Rome au Canada était un belge, le T. R. P. Dom Henri Smeulders, abbé mitré de l'ordre des Cisterciens, consulteur de la Sacrée Congrégation de la Propagande ». R. Rumilly, op. cit., 211. 
Mgr Smeulders ne pouvait mieux faire pour obtenir des réponses non favorables; était-ce son désir ? ${ }^{46}$

Mgr Smeulders quitte Montréal, en décembre 1884, sans avoir réglé les problèmes universitaires. ${ }^{47}$

A Montréal, la Succursale de l'Université Laval avait pour supérieur Thos.-E. Hamel, Grand vicaire de Québec. Si l'on en croit une lettre de ce personnage à Mgr A. Racine, le poste n'était pas une sinécure: c'était vivre dans le camp ennemi! Fatigué par le peu de résultats de sa présence à Montréal, accusé par les professeurs de l'Ecole de Médecine, menacé par les élèves en Droit, T.-E. Hamel consulte son ami de Sherbrooke:

Pensez-vous, Mgr, que je puisse tenir à ce régime ? Non, c'est plus fort que mon possible. Je vais endurer mon mal le reste de cette année, pour ne pas faire d'esclandre et pour ne pas augmenter les embarras. Vous savez, Monseigneur, que mon élection comme Supérieur (à la Succursale de Montréal) a été très mal vue à Rome. Mgr l'Archevêque m'a écrit dernièrement sous secret que le Cardinal Siméoni s'était plaint de ce qu'on m'avait désigné pour venir à Montréal cette année, et Mgr m'a demandé s'il n'y aurait pas moyen de changer cela et d'en mettre un autre... J'ai déjà fait ma résignation à l'Archevêque. ${ }^{48}$

Mgr de Sherbrooke approuve le supérieur de songer à quitter Montréal. Son sort est celui d'un général d'armée qui a tous ses soldats et tous ses officiers contre lui: «A ta place, j'insisterais pour un prompt rappel à Québec...; tu rendras plus de services à l'œuvre de l'Université, à Québec, qu'ici à Montréal. » ${ }^{49}$ Il ajoute même dans une lettre à Benj. Pâquet: «Sa présence à Montréal est nuisible à l'extrême et mal vue du Préfet et de l'Archevêque. Mgr Hamel doit revenir à Québec où il aurait dû rester. » ${ }^{50}$ Et $\mathrm{Mgr} B$. Pâquet de répondre avec tristesse: «Plus les événements marchent, plus je suis porté à

$46 \mathrm{Mgr}$ A. Racine à Mgr Taschereau, 8 avril 1884, Un. 141 - No D.

47 R. Rumilly, op. cit., 211-245.

48 T.-E. Hamel à Mgr A. Racine, 20 novembre 1884, AAS, XIII, A-1, F-1; Un. 143 - No BK; 143 - No CF.

$49 \mathrm{Mgr}$ A. Racine à T.-E. Hamel, 21 novembre 1884 , Un. 143 - No BM.

$50 \mathrm{Mgr}$ A. Racine à Benj. Pâquet, 21 novembre 1884, Un. 143 - No BO. 
croire que le bon Dieu ne nous veut pas à Montréal. » Sentiment imputable peut-être à quelque dépression nerveuse du Procureur du Séminaire de Québec qui écrit: «Je vous remercie des souhaits que vous faites pour le rétablissement de ma santé. Mais je crois que ... je suis un homme fini. $\gg^{51}$

Un peu de miel adoucira pourtant l'aigreur... Rome entreprend de décerner des honneurs ecclésiastiques. Il y en a pour tout le monde. L'Archevêque de Québec est élevé au Cardinalat en 1886; les évêques de Montréal et d'Ottawa deviennent archevêques; le Grand vicaire Hamel reçoit la nomination de Protonotaire apostolique;52 $\mathrm{Mgr}$ de Sherbrooke se voit attribuer le titre d' « assistant au trône pontifical ».53

Et ainsi, comme le dirait le Père Brichet, «facta est transquillitas magna ».

Le 28 décembre 1886, le nouvel archevêque de Montréal invite Mgr A. Racine à se rendre sur les lieux pour étudier les problèmes de Montréal et comprendre davantage la situation: «Connaître l'importance du poste sous le rapport religieux ». ${ }^{\mathbf{5}}$ Cette invitation de l'archevêque de Montréal, ce séjour d'étude "sur les lieux », gagneront-ils à la cause montréalaise le défenseur acharné des droits de Québec ? Il apparaît bien qu'un tournant peut se dessiner ici, dans la vie de Mgr A. Racine.

\section{Chaptrae VII}

\section{L'UNION DES DEUX ECOLES DE MEDECINE}

La volonté du Saint-Siège en créant la province ecclésiastique de Montréal - Désir de régler le problème universitaire Le partage des biens des Jésuites - L'abbé Jean-Baptiste Proulx, vice-recteur - Les préparatifs de l'union - Le vote du projet de loi à la Législature.

Une distribution d'honneurs ecclésiastiques avait sûrement contribué à dissiper l'atmosphère un peu chargée de l'assemblée F-1.

51 Benj. Pâquet à Mgr A. Racine, 24 novembre 1884, AAS, XIII, A-1,

52 Mgr A. Racine à T.-E. Hamel, 28 mars 1887, Un. 73, No 24.

53 Benj. Pâquet à Mgr A. Racine, 23 décembre 1886, AAS, XIII, B-1, A-3; Mgr Taschereau à Mgr A. Racine, 28 décembre 1886, AAS, XIII, B-1, A-3.

$54 \mathrm{Mgr}$ Fabre à Mgr A. Racine, 28 décembre 1886, AAS, XIII, B-1, A-3. 
des évêques de la Province de Québec. Toutefois, il faut chercher plus en profondeur la cause de l'accalmie qui va, cette fois, perdurer. Au Consistoire du 7 juin 1886, ${ }^{55}$ le Saint-Siège avait divisé l'ancien diocèse de $\mathrm{Mgr}$ de Laval en trois provinces ecclésiastiques: Québec, Montréal, Ottawa. Le 2 février 1889, Léon XIII, par une lettre aux évêques du Canada français, lettre où il déterminait le partage des biens des Jésuites, priait aussi les Evêques de la Province de Montréal de régler le problème de la Succursale de l'Université Laval.56

Dès janvier 1887, le supérieur du Séminaire de Montréal faisait savoir à $\mathrm{Mgr}$ de Sherbrooke, sa détermination d'affilier sa maison à l'Université Laval. Il entendait coopérer à l'établissement de la Faculté des arts à Montréal: «Le projet est arrêté, prêt pour l'exécution et approuvé par Monseigneur notre Archevêque. Nous avons même aujourd'hui l'espérance fondée que nos Commissaires d'école et le Gouvernement feront, de bon gré, passer leur Ecole Polytechnique... sous le contrôle catholique universitaire ${ }^{57} \mathrm{La}$ démarche avait plu au nouveau cardinal: "Vous avez vu que Laval gagne du terrain à Montréal. Il y a encore des arrangements qui sont en bonne voie. ${ }^{58}$ On semble donc s'acheminer résolument vers la concorde. On poussera même la bonne entente jusqu'à la délicatesse. Cette observation est de Mgr A. Racine:

Dans le Bill proposé «Acte pour incorporer le Syndicat », je remarque avec bonheur que le mot «Succursale » n'est pas mentionné, et c'est très bien fait. Je vous dirai en toute franchise qu'il faut éviter d'employer inutilement, un mot aussi impopulaire, qui sonne si mal aux oreilles des vieilles Montréalaises du District et de la Ville de Montréal. Ce mot

55 R. Rumilly, op. cit., 273.

56 Lettre de Léon XIII, Jam dudum, 2 février 1889, AAS, XIII, A-1, B-1; L'abbé Jean-Baptiste Proulx, vice-recteur de l'Université Laval à Montréal, à Mgr A. Racine, 26 novembre 1890: * Le Saint-Père a confié aux Evêques de la Province de Montréal le règlement de cette question », Collection Proulx, $\mathrm{II}^{\circ}$ vol., p. 116. Nous citerons désormais cette collection: Proulx, II : 116.

57 M. L. Colin à Mgr A. Racine, 8 janvier 1887, AAS, XIII, A-1, F-1. F-1.

$68 \mathrm{Mgr}$ Taschereau à Mgr A. Racine, 13 janvier 1887, AAS, X́III, A-1, 
nuisait à la bonne entente et ne servirait qu'à entretenir les préjugés qui existent déjà. Quant au paragraphe (a) dont je vous ai déjà parlé, il vaut mieux ne pas nommer Gouverneurs de droit, les Ev. de St-Hyacinthe et de Sherbrooke et laisser de côté aussi les futurs Evêques Suffragants, parmi lesquels pourraient fort bien se trouver quelques-uns des adversaires de Laval.59

Texte par où l'on voit le dessein de Mgr de Sherbrooke de supprimer toutes les causes de malentente. Un froissement se produisit pourtant par l'inégalité du partage des biens des Jésuites entre l'Université Laval $(\$ 100,000)$ et la Succursale de Montréal $(\$ 40,000) .^{60} \mathrm{Mgr}$ Benjamin Pâquet est d'ailleurs le premier à regretter le partage: «Je suis chagrin que la somme donnée à l'Université et à la Succursale, ne soit pas la même... Suivant moi, voilà une nouvelle source de misères. ${ }^{61} \mathrm{Mgr} \mathrm{A}$. Racine regrette, lui aussi, «que la Succursale ne reçoive que «\$40,000 piastres. Mais le $\mathrm{S}$. Père a décidé; donc c'est pour le mieux. " 62 Autant dire: cessons de nous quereller.

Par sa lettre de 1889, Léon XIII avait donné aux Évêques de la Province Ecclésiastique de Montréal, le pouvoir de nommer le vice-recteur de l'Université Laval à Montréal. Cet homme serait en pratique le Recteur de la Succursale. Au printemps de 1889 , la rumeur courait que le choix se porterait sur l'abbé Bruchesi. Aussitôt l'honorable J.-O. Chauveau presse Mgr A. Racine d'opposer son veto: "Maintenant on parle de l'abbé Bruchesi comme vice-recteur. Je crois qu'il serait très prudent de ne pas vous engager pour cette nomination. Il y a beaucoup d'objections. Je suppose que d'autres vous auront écrit dans ce sens. ${ }^{63}$

Le même jour, le vice-recteur du temps, l'abbé J. Marcoux, écrit à l'Évêque de Sherbrooke: «Je me propose d'aller vous rendre visite le plus tôt possible pour discuter avec Votre Gran-

$59 \mathrm{Mgr}$ A. Racíne à l'abbé Marcoux, vice-recteur de l'Université Laval à Montréal, 30 mars 1887, Un. 147.

60 Lettre de Léon XIII, Jam dudum, 2 février 1889, AAS, XIII, A-1, B-1; Mgr B. Pâquet à Mgr A. Racine, 21 février 1889, AAS, XIII, B-1, A-3.

61 Ibid.

62 Mgr Racine à Mgr B. Pâquet, 19 mars 1889, Un. 149.

63 J.-O. Chauveau à Mgr A. Racine, 21 mars 1889 , AAS, XIII, B-1, A-3. 
deur la situation présente et prendre vos conseils. ${ }^{64}$ Le 21 juillet de la même année, le conseil de l'Université Laval, avec l'approbation des évêques de Montréal, nommait vice-recteur de l'Université Laval à Montréal, non l'abbé Bruchesi, mais l'abbé Jean-Baptiste Proulx, curé de St-Lin des Laurentides. ${ }^{65} \mathrm{Mgr}$ A. Racine s'en félicite: «Je crois que le choix est excellent pour Québec et pour Montréal. » ${ }^{66}$

On songe donc sérieusement, en cet été 1889 , à «l'absorption de l'École de Médecine et de Chirurgie de Montréal par Laval ». Terme même dont se sert Mgr B. Pâquet, qui voit là une affaire très grave, laquelle selon lui, ne saurait être réglée pour le 2 octobre, jour de l'ouverture des classes à l'Université. ${ }^{67}$ Monsieur le vice-recteur est à ce moment-là à Québec pour discuter le problème; il fait la rencontre de Mgr de Sherbrooke alors de passage dans la vieille capitale. "Je lui exposai, comme à un homme de bon conseil, plein de confiance dans sa longue expérience, l'embarras dans lequel nous nous trouvions à Montréal. ${ }^{68}$ Mgr A. Racine lui glisse ce conseil:

- Pourquoi n'ouvrez-vous pas les cours avec les deux Ecoles réunies, quitte ensuite à vous en rapporter à ce que Rome aura décidé ?

- Je le ferai volontiers, mais, je le comprends, c'est un moyen extrême, pénible; Monseigneur Pâquet n'y consentira jamais.

- Pas du tout. Il vient de me dire que vous pouviez ouvrir, au jour fixé.

- Oui, ouvrir avec Laval seulement, mais pas avec l'Union; et il m'a affirmé qu'il vous l'avait dit.

- Monseigneur, il doit y avoir un malentendu quelque part.

64 L'abbé J. Marcoux à Mgr A. Racine, 21 mars 1889, AAS, XIII, B-1,

65 Proulx, I, 11 et 14; Mgr B. Pâquet à Mgr Racine, 26 juillet 1889, AAS, XIII, B-1, A-3; l'abbé L. Colin à Mgr Racine, 27 août 1889, AAS, XIII, B-1, A-3.

${ }_{66}$ Mgr A. Racine à Mgr B. Pâquet, 23 juillet 1889, Un. 150.

67 Mgr B. Pâquet à l'abbé Proulx, 25 septembre 1889, Proulx I: 92.

$68 \mathrm{La}$ 《 note » qui fait suite au document précédent, contient la citation que nous venons de faire ainsi que la conversation entre Mgr A. Racine et l'abbé Proulx. 
Lequel des deux a faussé la vérité ? Mgr Racine ou Mgr Pâquet? L'abbé Proulx commence «à marcher sur des lames de rasoir ${ }^{69}$ Il se rend immédiatement chez Mgr Pâquet pour exiger des explications: "Mgr Pâquet m'a dit que je pouvais ouvrir avec Laval, mais aucunement avec l'Union... Je m'empresse de vous écrire cette rectification, car ce malentendu m'a été bien pénible ${ }^{70}$ écrit-il sur-le-champ à Mgr A. Racine qui a quitté Québec pour Sherbrooke aussitôt après l'entretien mentionné plus haut. Or, cette lettre à peine mise à la poste, $\mathrm{Mgr}$ Pâquet rencontre l'abbé Proulx et lui dit:

A propos, je me rappelle; en effet j'ai dit à $\mathrm{Mgr}$ Racine, sur les représentations qu'il m'a faites, que vous pouviez, si vous le vouliez, ouvrir les cours avec l'union des deux Ecoles de Médecine, à vos risques et périls, quitte à en passer par ce que Rome déciderait.

Aussitôt, l'abbé Proulx lui demande de mettre le propos par écrit. A quoi Mgr Pâquet lui répond: "Bien loin de là, il m'est impossible de vous le permettre officiellement, et de vous le conseiller. ${ }^{71}$

Ces petits détails peignent bien une situation délicate où le vice-recteur, mal en point, marche «vers l'inconnu, au milieu de réticences habiles et de réserves prudentes ».

Dans sa lettre du 12 juin 1890, le Cardinal Siméoni, Préfet de la Congrégation de la Propagande, avait employé des exhortations fermes en vue d'un prompt règlement:

Ce n'est point une question à juger par des impressions de parti, mais par des vues de bien public plus élevées et plus sereines...

C'est pourquoi je prie Votre Grandeur ( Mgr Chs Fabre) et avec Elle les Evêques Suffragants, d'user de tout ce que vous avez de prudence et de sagesse, de sollicitude et d'autorité, afin d'écarter les sujets de discussion et de litige, de calmer les esprits et de faire, qu'en vue du plus grand bien, et sous la ré-

69 Proulx, I: 96.

70 L'abbé Proulx à Mgr Racine, 26 septembre 1889, Proulx, I: 94.

71 Proulx, I: 96.

72 Le Le Cardinal Siméoni aux Evêques de Montréal, St-Hyacinthe et Sherbrooke, 12 juin 1890, Proulx, X: 234; AAS, XIII, B-1, A-3. 
serve des droits susdits, on s'entende de part et d'autre pour réaliser l'union et pour accepter de grand cœur les sacrifices exigés par une œuvre si utile.

Mgr Racine eut souhaité une lettre circulaire des trois Évêques de la Province Ecclésiastique de Montréal par laquelle ils eussent donné à tous leurs diocésains, connaissance $1^{\circ}$ de la lettre du Saint-Siège à l'archevêque de Montréal et à ses suffragants $; 2^{\circ}$ du document récemment signé par eux pour la formation d'un comité en vue de s'entendre avec l'École de Médecine de Montréal; $3^{\circ}$ du désir de tous d'obéir aux volontés du SaintSiège, et donc appel à la bonne volonté de tous les gens de bien, qui aiment leur religion et leur pays. ${ }^{73}$

Le projet d'union comprenait deux articles essentiels relatifs à l'École Victoria: premièrement, cette École de Médecine et de Chirurgie de Montréal deviendrait la Faculté de Médecine de l'Université de Montréal régie d'après la charte amendée; deuxièmement, les biens possédés par les anciens membres de l'École de Médecine resteraient leur propriété; ils pourraient se les partager entre eux ou les passer à la nouvelle Faculté de Médecine de l'Université. L'accord se fit entre les membres des deux comités formés pour consommer l'union des deux Écoles de Médecine. $^{74} \mathrm{La}$ nouvelle réjouit $\mathrm{Mgr}$ Racine: "Je suis heureux d'apprendre que l'union prend une bonne tournure. ${ }^{75}$ Une dizaine de jours après $\mathrm{Mgr}$ de Sherbrooke se rend à Montréal pour rencontrer les deux comités de coordination et prendre part aux délibérations. Il a dû se montrer bien conciliant, vu que l'abbé Proulx lui écrit le lendemain: «Les médecins de l'École ont été satisfaits de notre entrevue d'hier soir. Vous les avez gagnés tout à fait par vos manières de faire, franches et larges. ${ }^{76}$ On est même porté à croire que Mgr A. Racine a dirigé

$73 \mathrm{Mgr}$ A. Racine à L.-D.-A. Maréchal, administrateur du diocèse de Montréal en l'absence de Mgr Fabre, en voyage à Rome, 3 septembre 1890 , Proulx II : 12.

74 Proulx II : 28 et 29. L'abbé Proulx à Mgr A. Racine, 18 octobre 1890, Proulx II : 27.

$75 \mathrm{Mgr}$ A. Racine à l'abbé Proulx, 18 octobre 1890, Proulx II: 27.

76 L'abbé Proulx à Mgr A. Racine, 28 octobre 1890, Proulx II : 42. 
la discussion, puisque le vice-recteur se met à rédiger «ce document important dont vous m'avez passé les idées ${ }^{77}$

Il ne reste plus qu'à s'adresser à la Législature Provinciale pour en obtenir l'amendement indispensable de la charte. Mais auparavant on se souvient que l'Évêque de Sherbrooke propose à ses confrères, les évêques de la Province de Montréal, de préparer les esprits par une lettre circulaire commune. Cette lettre paraît le 8 novembre $1890 .^{78}$ La même journée, les mêmes personnages écrivent une seconde lettre collective adressée cette fois au Premier Ministre Honoré Mercier. "L'entente a fait de tels progrès qu'il dépend désormais d'une seule condition, que cette union si désirable passe à l'état de fait accompli: il suffit que la sanction législative soit donnée... pour l'amendement de la charte de l'École de Médecine et de Chirurgie de Montréal. »79 Lettre qui ne manque pas d'habileté: après avoir énuméré les raisons qui militent en faveur de l'union, soit le bien de la religion catholique et le bien du public, les Évêques continuent: "Nous n'insisterons donc pas sur l'honneur qui reviendrait à votre gouvernement d'assurer le succès d'une affaire aussi épineuse qu'importante, ni sur la grandeur du service que vous rendriez ainsi à notre province. ${ }^{80}$ Affaire, disait-on, qui ne soulèvera que des oppositions de mauvaise volonté qui tomberont d'elles-mêmes «quand vous aurez élevé... la question à la hauteur qui lui appartient. $\gg 81$

Mercier hésite devant les dangers de querelle. Il promet d'agir, mais à la condition - pour se couvrir, sans doute - qu'on lui présente un ordre exprès du Saint-Siège..$^{82}$ Rome se fait attendre, en dépit des deux télégrammes envoyés à Mgr Jacobini pour engager le Cardinal Siméoni à écrire ou à télégraphier au Premier Ministre. ${ }^{83}$ Mgr Fabre se trouve alors à Rome pour

77 Ibid.

78 Nous donnerons en appendice $V$, à la fin du présent ouvrage, le texte complet de cette lettre circulaire. Proulx, $\mathrm{X}: 234$.

79 Lettre collective des Evêques de la Prov. eccl. de Montréal à l'Honorable H. Mercier, 8 novembre 1890 , Proulx, II : 62 .

80 Ibid. 64.

81 Ibid. 65.

82 L'abbé Proulx à Mgr Racine, 14 novembre 1890, Proulx, II: 72.

83 Ibid., 68. 
presser les affaires. ${ }^{84}$ Mgr Jacobini envoie deux télégrammes, l'un à l'abbé Proulx: "Télégraphié Mercier attendre lettre», l'autre à l'Honorable Premier Ministre: «Attendre lettre sur bill Union Ecole $\$^{85}$

Tout semble bien aller. La lettre de Rome, espère-t-on, sera favorable. ${ }^{86}$ Le malheur est que tous ces arrangements s'élaborent à l'insu du Conseil de l'Université Laval. Des protestations sont à prévoir qui ne manqueront pas:

Proposé par Mgr le Recteur et résolu que, l'Université Laval, après tous les bons procédés dont elle a usé envers sa succursale de Montréal, après tous les sacrifices qu'elle s'est imposés pour lui donner et lui continuer l'existence, est grandement surprise et peinée de voir que, dans une question aussi grave que celle dont il s'agit dans la loi proposée (Loi de l'Ecole de Médecine), le Conseil Universitaire n'ait pas été prévenu et n'ait pas eu l'occasion de donner son avis. ${ }^{87}$

Tout le monde, à Montréal, s'attendait à ce genre de difficultés. Mgr Racine, le premier, avait écrit: "Cependant il faut marcher de l'avant... Il ne faudra point pour aucune raison laisser entamer les points fondamentaux de l'Union, tels qu'exprimer dans le Bill. ${ }^{88}$

Le 20 novembre, à quatre heures de l'après-midi, note le vice-recteur, je recevais le câblogramme de Rome signé par Mgr Jacobini et adressé en réalité à l'Honorable Premier Ministre, en vue de l'engager à sanctionner l'amendement à la Charte de l'Université pour l'union à Montréal des deux Écoles de Médecine. ${ }^{89}$

Le projet de loi est donc présenté à la Chambre le même jour, puis en deuxième lecture le 23 novembre, pour ensuite subir l'épreuve du comité des bills privés: "C'est le passage de la

84 Ibid., 68.

85 Ibid., 73.

86 Ibid., 74.

87 Extrait du registre des délibérations du Conseil Universitaire, en date du 17 novembre 1890. Copie envoyé par le Cardinal Taschereau à Mgr A. Racine, AAS, XIII, B-1, A-3; Mgr B. Pâquet à Mgr A. Racine, 22 novembre 1890 , ibid.

$88 \mathrm{Mgr}$ A. Racine à l'abbé Proulx, 17 novembre, Proulx, X: 251.

89 Proulx, II : 86, 87, 88. 
mer Rouge. ${ }^{90}$ L'évêque de Sherbrooke répond à son ami Proulx: "Je vous souhaite un passage de la Mer Rouge aussi heureux que celui de Moïse; tout de même, soyez sur vos gardes, car l'armée de Pharaons vous suit de près. ${ }^{91}$ Le vice-recteur le sent bien :

Je ne vous cache pas qu'il est rumeur d'une opposition du Conseil Universitaire; j'espère toujours qu'on nous épargnera ce scandale. J'ai écrit une lettre à Son Eminence pour le supplier d'user de son influence pour qu'on ne donne pas, soit dans l'Université catholique, soit parmi les amis de l'Université catholique, un pareil coup à l'autorité des évêques et au respect dû aux désirs du Saint-Père. ${ }^{92}$

Le 29 novembre, la Chambre suspend par privilège le cours ordinaire de la Session pour discuter le projet de loi de l'union; il est voté en troisième lecture, aussitôt le rapport du comité rédigé. ${ }^{93}$ Comme on pouvait s'y attendre, la chose blesse profondément les administrateurs de l'Université. Mais nous reviendrons sur ce sujet au prochain chapitre.

La cause de Montréal est gagnée; il ne reste plus qu'à s'offrir de part et d'autre, des félicitations. On n'y manque pas. L'abbé Proulx y va de sa lourde plume: "A vous, Messeigneurs, mes remerciements et mes humbles félicitations pour la prudence et la fermeté dont vous avez fait preuve dans cette circonstance délicate, suaviter et fortiter. ${ }^{94}$

Mgr A. Racine félicite le vice-recteur de sa prudence, de son habileté et de son dévouement:

Je vous remercie de tout cœur de ce que vous avez fait pour l'union des deux Ecoles de Médecine de Montréal; vous avez réussi au-delà de toute espérance, malgré les obstacles et les difficultés qui surgissaient de tous côtés. Ainsi je vous offre mes plus sincères félicitations; vous avez travaillé «sicut bonus miles Christi ».

\author{
GeRMAIN LAVALLÉE, ptre, \\ Sherbrooke, P. Q. \\ (à suivre)
}

90 L'abbé Proulx à Mgr A. Racine, 21 novembre 1890, Proulx, II : 98. $91 \mathrm{Mgr}$ A. Racine à l'abbé Proulx, 23 novembre 1890, Proulx, X : 252. 92 L'abbé Proulx à Mgr Racine, 26 novembre 1890, Proulx, II : 116. 93 Proulx, II : 119.

94 L'abbé Proulx à Mgr A. Racine, 5 décembre 1890, Proulx, II : 142. 